\title{
Multi-Fractal Model of Coal Resources Distribution and Its Application in Spatial Distribution Law of Coal thickness
}

\author{
Liu Xing*, Hu Baolin and Jiang Songmei \\ Anhui University of Science and Technology, 232001, HuaiNan, \\ Anhui Province, China \\ liuxing0795@126.com
}

\begin{abstract}
Most study on the coal thickness distribution mainly focus on geology analysis by geophysical data currently, some researchers apply trend surface method to extract hidden information from those geological data to reveal the law of coal thickness distribution effectively. This paper proves that the coal resources and coal thickness distribution follows fractal and multi-fractal model based on the principle of coal depositing, and demonstrates the fractal characters of global coal resources and China coal resources by the statistical data. Further more, this study utilizes 168 coal thickness of M10 from Huaibei coal field through dills to separate the background and abnormity map by $S$-A method with some necessary pre-processing, the resulted background map can be looked as a trend map of trend surface analysis and the abnormity map is as residual map according to the mathematical model, the contour map of background shows the deposition rule that the thick coal distributes around the underlying sand with shallow water and the thin coals are on the thick sand and split bay with stronger spatial corresponding relationship, while the abnormity map shows the secondary coal thickness change is related to the process of filling and level up, the location is also coincident with the underlying deposit environment. The result by $S$-A method reveals the coal thickness distribution more clearly and precisely and more close to the deposition rule contrasted with the result by trend surface analysis, it is concluded that the coal thickness distribution follows the multi-fractal model and the S-A method is effective for those geological data processing.
\end{abstract}

Keywords: coal thickness, spatial distribution, multi-fractal, S-A filter, self-similarity

\section{Introduction}

The spatial distribution of most ore deposits(resources) can be modeled by grade-tonnage and multi-fractal method quantitatively [1-2]. But there is little research in the coal resources spatial distribution law, it is significant to find out the spatial distribution principle of coal resources because the coal occupies an important position in the state economy, especially essential for coal mine design and mining itself for that the coal thickness is related to the mining deployment and recovery rate. The study on the coal thickness spatial distribution and the change regularity provides strong geology safeguard for coal mine production activities and brings huge economic benefits. Meanwhile, the correct understanding of coal thickness and its distribution rule is the basis of mining method determination and the theoretical basis of reserve estimation [3-4]. The popular ways for coal thickness change research are divided into two classes: mathematic geology and detecting [5-6]. The mathematic way is to find out the law of coal thickness change by mathematical model on the base of existing data, which owns the advantage of low cost and can be extended to other coal filed for application. The

* *Corresponding Author 
trend surface analysis which is one of the mathematical geology methods is widely employed in the coal thickness change analysis currently.

The process of coal accumulation has the typical characteristics of self-organizing, criticality and spatio-temporal singularity [1]; it means that the coal forms spontaneously with ordered structure in space and in history without of outside interference [7] in other words. Being similar to other geological process of mineralization, the distribution of coal resources and its thickness is influenced by the earth's inner nonlinear drive and exterior depositional environment, with the characteristics of huge energy release and matter enrichment in short time and in narrow space [6],the abnormity of energy release and matter enrichment is called singularity[6].The singularity, self-similarity and criticality may be the basal characteristic of result caused by nonlinear and complex geological process, the result could be measured and studied by fractal and multi-fractal model [10-13].

This paper demonstrates the fractal characteristic of global coal resources and China coal resources by the pressed data and map firstly, and then draws the log-log plot of area( $>=h)$-thickness to show multi-fractal pattern existing in the coal field which forms in the river-delta environment, and decompose the coal thickness map by S-A method into background and abnormity map. The background map is contrasted with the underlying sandstone map to find out the law of coal distribution, and the advantage of S-A method is drawn when compared with the classical trend surface analysis method.

\section{The Fractal Characteristic of Coal Resources Spatial Distribution}

\subsection{The Spatial Distribution Pattern of Depositional Process and Sediment}

Many studies imply that the multi-fractal model is a common pattern widely existing in the sediment shape, grain size, physical property, thickness distribution over space and time no matter modern or ancient one [5,9]. In literature 17, the author studied the depositional law induced by the difference of rock grains transporting leading by hydrodynamic change, deduced the multi-fractal relationship between the amount(mass) and grain size, and classified the rock types for engineering mechanics purpose by the fractal dimension; in the area of depositional process and it's record, literature 18 indicates that the sediment thickness has the self-similarity by studying the series of mud sediment sequence and sediment interface, and the fractal dimension passes from the process to the sequence, it is a way to understand the law of sediment sequence and its accumulation.

The intrinsic of coal accumulation is some like other depositional minerals, influences by the ancient topography, sand supply, climate and crust unbalanced subsidence, directly controlled by eustacy, river channel change and local marsh evolvement [7-8], the shape and thickness of coal accumulation is processes of multiplicative cascade, which is applied to explain many physical process leading to continual multi fracture [6]. The superposed accumulation of coal could be described by this model, firstly, suppose that there is a limited room called accumulation unit, and suppose that there are a series of depositional events occupied in this room further, the sediments distribution of different accumulation stage are similar in thickness and shape at space, the continuous accumulation may result in the enrichment or thin in the same area, if the accumulated thickness of coal reaches the economical limit then called commercial seam [9]. If taking the product of accumulation strength and the accumulation range as an constant, the last coal resources distribution by multi-stage accumulation could be represented by multiplicative cascade process. Writing $h(\varepsilon, X)$ for the coal thickness, $X$ is the position, $\varepsilon$ is a radius around $X$,the range and location of coal accumulation change depending on the crust unbalanced subsidence, resulting in enrichment or thin of the coal seam and maybe some retained locally, then the change law of coal thickness distribution 
is described by this equation: $h\left(\varepsilon_{n}, X\right)=\lambda(X) h\left(\varepsilon_{n-1}, X\right), \varepsilon_{n}=\beta(X) \varepsilon_{n-1}$, here, $h\left(\varepsilon_{n-1}, X\right)$ is the coal thickness distribution before current accumulation, $\lambda(X)$ is a coefficient about coal accumulation intensity, and $\beta(X)$ is the rate of range change at current location, simultaneous equations are:

$$
\begin{gathered}
\frac{h\left(\varepsilon_{n}, X\right)}{\varepsilon_{n}} \times \frac{\varepsilon_{n-1}}{h\left(\varepsilon_{n-1}, X\right)}=\frac{\lambda(X)}{\beta(X)} \\
\frac{\ln \lambda(X)}{\ln \beta(X)}=\frac{\ln h\left(\varepsilon_{n}, X\right)-\ln h\left(\varepsilon_{n-1}, X\right)}{\ln \varepsilon_{n}-\ln \varepsilon_{n-1}}=\frac{\partial \ln h}{\partial \ln \varepsilon} \\
\frac{\ln \lambda(X)}{\ln \beta(X)} \times \frac{h}{\varepsilon}=\frac{\partial h}{\partial \varepsilon}
\end{gathered}
$$

The coefficient $\ln \lambda(X) / \ln \beta(X)$ is looked as coal accumulation intensity at $X$, the coal thickness becomes uneven after a number of times depositional events in one unit, intergrading equation (2):

$$
\begin{gathered}
\int \frac{\partial \ln h}{\partial \ln \varepsilon} d \ln \varepsilon=\int \frac{\ln \lambda}{\ln \beta} d \ln \varepsilon \\
\ln h=\ln \varepsilon \times \frac{\ln \lambda}{\ln \beta}=\ln \varepsilon^{\frac{\ln \lambda}{\ln \beta}} \\
h=\varepsilon^{\frac{\ln \lambda}{\ln \beta}} \\
h=k \times \varepsilon^{\alpha}+c
\end{gathered}
$$

So, the average coal thickness of a unit is power-law relation to the size of unit, the exponent $\alpha$ is not a constant and maybe multi-value due to the different distinct accumulation events, showing that the coal thickness follows multi-fractal distribution obeying a process of multiplicative cascade.

Moreover, Cheng [6] proves the fractal and multi-fractal model existing in "gene map" such as Fourier spectrum, eigenvector spectrum of original data, and the mixing pattern can be decomposed into background and abnormity part by principle of generalization self-similarity [1].

\subsection{The Fractal Characteristic of Coal Resources Distribution}

The global map of coal resources distribution is digitalized to topological polygon map by ARCGIS, and take the areas of coal fields to represent theirs resources in reason because the map is large-scale bedded deposit and the larger the area the abundance the coal resources generally. The relative coordinate unit has scale meaning, 156 coal fields is recognized on the map with the largest area of 824.335 and the least area of 1.407 relative. Sort the coal fields by area, count the number of each group by $N(\geq A), A=0,10,50,100,500$; the number of each group and the area satisfies the following formula:

$$
N(\geq A) \propto A^{-D} \quad \ln N(\geq A)=C-D \ln A
$$

Plot the logarithmic numbers and logarithmic area of each group on rectangle coordinate system, and fit the points by the least square method; the correlation coefficient is obtained at the same time. The original map and the result data are demonstrated in Table 1 and Figure1, Figure2. 


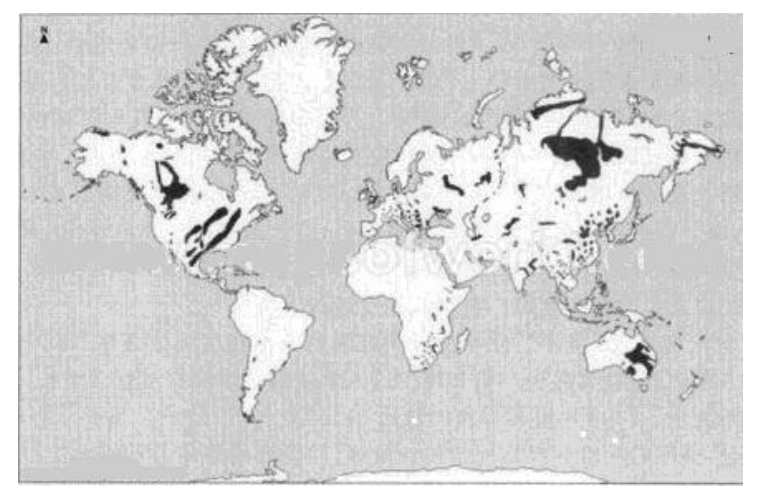

Figure 1. The Map of Global Coal Distribution

Table 1. The Statistical Area of Global Coal Fields

\begin{tabular}{|c|c|}
\hline$>=$ area(relative) & Number \\
\hline 1 & 93 \\
\hline 10 & 47 \\
\hline 50 & 9 \\
\hline 100 & 6 \\
\hline 500 & 1 \\
\hline
\end{tabular}



Figure 2. The Log-Log Plot of Number and Area

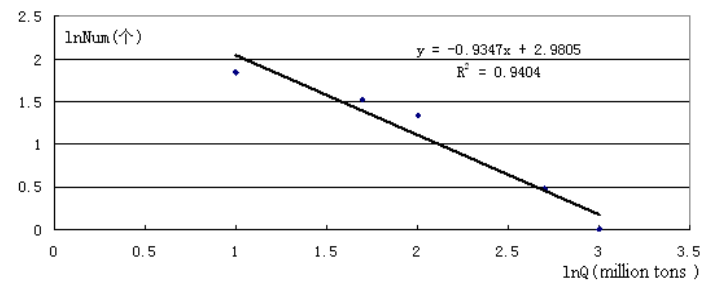

Figure 3. The Log-Log Plot of Number and Coal Resources

Table 2. The Resources of Main Coal Fields in China

\begin{tabular}{|l|r|l|r|l|r|}
\hline name & resources $\left(10^{8} \mathrm{t}\right)$ & name & resources $\left(10^{8} \mathrm{t}\right)$ & name & resources $\left(10^{8} \mathrm{t}\right)$ \\
\hline Datong & 373 & Tengzhou & 37 & Hegang & 20 \\
\hline Ningwu & 412 & Yanzhou & 33 & Dayan & 36 \\
\hline Xishan & 193 & Jining & 32 & Baorixile & 41 \\
\hline Huoxi & 309 & Juye & 55 & Hushan & 23 \\
\hline Qinsui & 843 & Huanghebei & 25 & Yimin & 48 \\
\hline Hedong & 515 & Longkou & 27 & Yiminwumuchang & 53 \\
\hline Jiaozuo & 24 & Hanqing & 57 & Honghuaerji & 27 \\
\hline Xinmi & 27 & Weixian & 14 & Huheruoer & 104 \\
\hline Yuzhou & 17 & Kailuan & 43 & Zhalairuoer & 104 \\
\hline Pingdingshan & 50 & Jingxi & 20 & Huolinhe & 131 \\
\hline Yingxia & 25 & Tiefa & 19 & Wunite & 69 \\
\hline Huainan & 259 & Jixi & 23 & Baiyinhua & 140 \\
\hline Huaibei & 67 & Qitaihe & 17 & Shengli & 214 \\
\hline
\end{tabular}




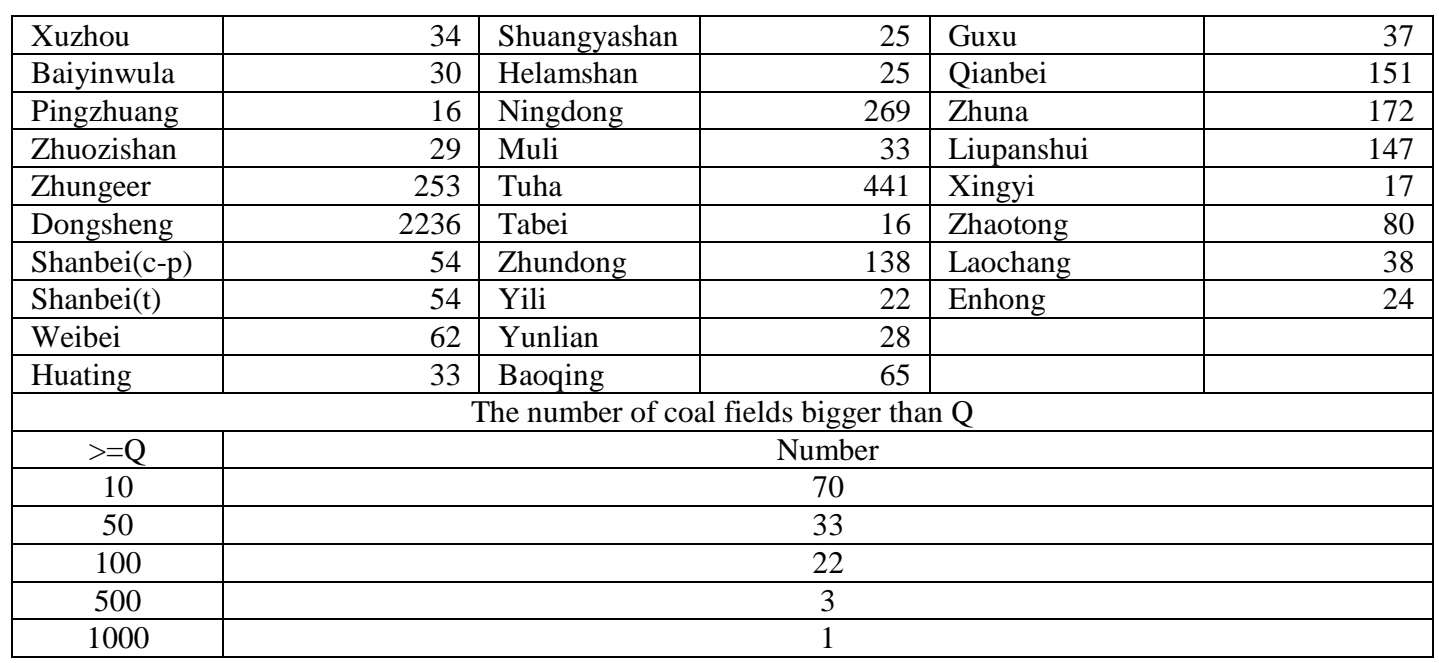

The coal resources quantity of China are from pressed literatures till year 2011, the numbers of coal fields with resources bigger than 10, 50, 100, 500, 1000 hundred tons are counted separately which meets the formula: $N(>=Q) \propto Q^{-D}$ and illustrates in Figure 3, indicating the linear relationship between the logarithm of coal fields number and their quantities. The fractal dimension $\mathrm{D}=0.9347$, and correlation coefficient is 0.9404 showing good fitness.

The data and Figures above indicate that not only the coal resource spatial distribution but also the coal resource quantities follow fractal distribution; the fractal dimension of global is 0.7338 less than that of China of 0.9347 , implying the fact that the coal resources distribution of China is more uneven than that of global.

\section{The Study of Coal Thickness Distribution Based on S-A Method}

\subsection{Data Description}

The original data for study is from 168 boreholes in Luling coal field of Huaibei, China, the thickness of No.10(M10) coal of every boreholes is extracted and the sand thickness underlying and overlying M10 is also recorded for study. Luling coal field is located at south-east of Huaibei coal field, the full field is about 9 kilometers long from north to south and about 5 kilometers wide from east to west. The M10 deposits in the river-delta plain environment [9], the law of coal thickness distribution is an important focus for coal mining, the relationship between the underlying sandstone and the coal distribution is essential for understanding the sedimentary evolution but which is still in discussion through traditional ways. The coal thickness and sand thickness are adjusted to vertical thickness; the distortion of coal is ignored for the inexistence of strong structure, the compact for the coal seam is even for the close burying depth, and there is no magma intrusion existing.

\subsection{The Statistics and Pre-Processing of Data}

The statistics of M10 thickness from 168 boreholes are list in Table 3, its variation coefficient is $\mathbf{5 5 . 8 \%}$ indicating the biggish variability in the data, Q-Q plot (Figure 4a) also denotes the data deviating reference line of Gaussian distribution, the significant index of K-S normal testis is less than 0.01 .

The S-A method employed in this study later needs gridded data, while the kriging method of data gridding runs perfectly when the data satisfies normal distribution or closes to normal distribution on the hypothesis of two order stationary ${ }^{[3]}$. The S-A method is good at outliers processing without requirement of data distribution, so the data of M10 thickness is transformed by Box-Cox method, the transformed data satisfies normal 
distribution approximately but some outliers are still reserved(the outliers follows fractal distribution actually) (Figure 4b), and then carry on krigging method to grid the data, the contour map of M10 thickness based on gridded data is in Figure 5a.

Table 3. Statistics of M10 Thickness

\begin{tabular}{cllll|lll}
\hline M10 Thickness & mean & middle & $\min$ & $\max$ & Standard deviation & $\begin{array}{l}\text { coefficient } \\
\text { of variation }\end{array}$ \\
\hline \multirow{4}{*}{ meter } & & & & & & $55.8 \%$ \\
\cline { 2 - 7 } & 2.5 & 2.46 & 0 & 12.6 & 1.45 & Transformed data \\
\cline { 2 - 7 } & \multicolumn{3}{c}{ Original data } & \multicolumn{3}{c}{ Sig } \\
\cline { 2 - 7 } & skewness & kurtosis & Sig & skewness & kurtosis & 1.0 \\
\hline
\end{tabular}

\subsection{Decomposition of Coal Thickness Map by S-A}

This study employs S-A model proposed by Cheng et al. [6] to decompose the coal deposit pattern. The S-A model has been proved as a powerful method for geochemical anomalies extraction from background in different geochemical field including stream sediments and lithogeochemical data [6], which is based on scale invariance quantification in frequency domain and characterizes density-area relationship of the spectral energy [2], It can be written by the following equation:

$$
A(\geq s) \propto s^{-d}
$$

Here $S$ denotes the value of spectral energy density, A $(\geq S)$ denotes the area with a threshold greater than or equal to $\mathrm{S}$, and $\mathrm{d}$ is related to the elliptical dimension, corresponding to isotropic dimension, the exponent of the power law and the symbol $\propto$ denoting proportionality. Carrying out of the S-A model has these steps: (1) Transform the gridded data into frequency domain using the fast Fourier transformation. (2) Calculate the power spectrum of gridded data. (3) Calculate $A(\geq S)$ the area with a threshold greater than or equal to S. (4) Draw $\log -\log$ plot of S-A, and fit the points by the least square method. (5) Choosing threshold value of $S$ (breakpoints in the log-log plot) and then construct the filter. (6) Inverse fast Fourier transformation of the resulted power spectrums. In step 5, there may be two or more filters. Usually, low and high pass filters constructed by choosing suitable breakpoint and therefore there are two resulted maps, the first is for the anomaly map and second is for the background map. In the current paper, these steps have been done with IDL Software.

\subsection{Result Contrast and Analysis}

Being like other physical and chemical signal of geological event, the thickness of coal is also one of physical signal which records the ancient geological process and usually has the self-similarity [6]. As the combined result of a good many geology factors, the superpose of climate change, mass supply, ancient topography, river shifting lead to the multi-fractal characteristic of coal distributes [9] in space and in history. The log-log plot of coal thickness $(\mathrm{h})$ and corresponding area $(>=h)$ is illustrated in Figure 6 to show the spatial multi-fractal pattern.

Based on the general self-similarity principle, the gridded coal thickness of M10 as a field is transformed into frequency domain to calculate the area-density of power spectrum in Figure 7, there are three straight lines fitted by means of the least squares method on log-log paper, Different slopes of the straight lines correspond to different sets of similarly power. The power that fall on three straight lines can be interpreted as different accumulation process. And three separate power-law relations between area and density indicate that power spectrum satisfy a multi-fractal model. The thresholds in slope on the power-law graph can be used to distinguish groups of samples with different statistical characteristics on the coal thickness map which corresponds to different 
populations. The observed value of coal thickness is composite of three components in fact:

$$
Z_{i}=B_{i}+A_{i}+R_{i}
$$

Where $Z_{i}$ denotes the observed value of coal thickness from borehole, $B_{i}$ is the accumulated coal thickness caused by A/S(accommodation /supply) change related to regional depositional background which can be represented by ancient topography or sedimentary facies, ${ }_{i}$ is usually seemed as locally accumulation of coal which is leaded by large-scale subsidence, stream transfer evolution, $R_{i}$ denotes the random noise in the signal. The erosion of later streams, structure compaction and magma intrusion may change thickness also but those can be looked as parts of ${ }^{A_{i}}$. The three components are

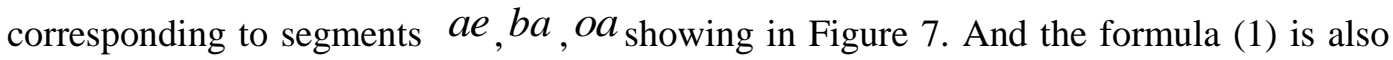
the principle of trend surface analysis, which is widely applied in the study of coal thickness distribution [5].

In Figure7, segment $a e$ corresponds to $B_{i}, b a$ is related to $A_{i}, o b$ represents $R_{i}$. They characterize different self-similarity at different non-scale range. The coal thickness of M10 is decomposed into background and abnormity maps (Figure 8a, Figure $8 b)$ by the S-A filter which is irregular in shape and good at keeping the anisotropy and inner structure of original map.

Figure 5a is the gridded contour map of M10 coal thickness by krigging, showing the thick coal occupying the right half part map from north to south and a rich centre at the low-left of map. Figure $5 \mathrm{~b}$ demonstrates the underlying sandstone distribution which represents the sedimentary environment just before M10, there is some direct relations between them based on the above analysis, but unexpectedly, the underlying sandstone indicates a channel existing from northwest to southeast with interdistributary bay and floodplain around. It is hard to relate the M10 coal thickness to the underlying sandstones in space. But the Figure 8a which is separated from Figure 5a by S-A shows good corresponding relationship with the Figure $5 \mathrm{~b}$ which denotes the ancient depositional environment, where the thick coal in the left-low part of Figure 8a is just on a shallow flood plain of Figure 5b, and the main thick coal distributes closely to those channel sand in concentric rings shape where the crevasse and shallow flood plain facies are usually developed. The rule of the thicker the sandstone the thinner the coal is clearly showing by contrasting centre-right Figure $5 \mathrm{~b}$ to Figure $8 \mathrm{a}$, the deeper the water the thinner the coal is also existing in the left part from Figure $5 \mathrm{~b}$ to Figure $8 \mathrm{a}$, and the inter-distributary bays between sandstone are the main room for coal accumulation, such as the two thick coal strips in the top the Figure 8a are corresponds to the inter-distributary bays in Figure 5b, the thick coal strips from west to east in Figure 8a is also upon the inter-distributary in Figure 5b. The thin coal strips of M10 are mainly located at the thicker sandstone and deeper water such as the centre and the left-top in Figure 5b and thinning out towards to the deep water basin gradually.

The Figure $8 \mathrm{~b}$ is looked as the later accumulation process by random events such as paleo-channels wriggling which might be the results of crust subsidence, climate change or mass supplying, it reflects the secondary change of coal thickness from the view of formula(1),posses the similar function as residual map of trendy surface analysis. The secondary change of M10 distributes mainly from northwest to southeast and from southwest to northeast in Figure 8b corresponding to the low land of deep flood plain in Figure $5 \mathrm{~b}$, which satisfies the depositional law. 


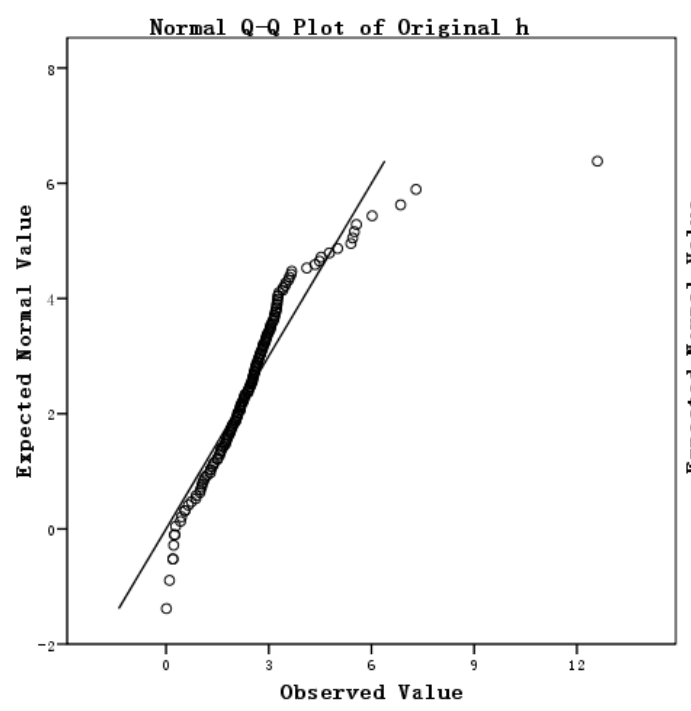

(a)

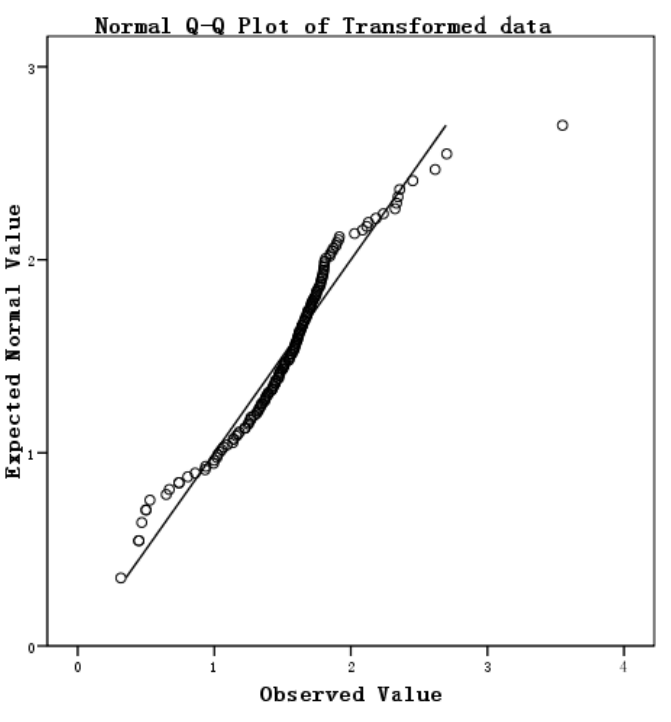

(b)

Figure 4. a: Original Q-Q Plot of M10 Thickness b: Transformed Q-Q Plot of M10 Thickness

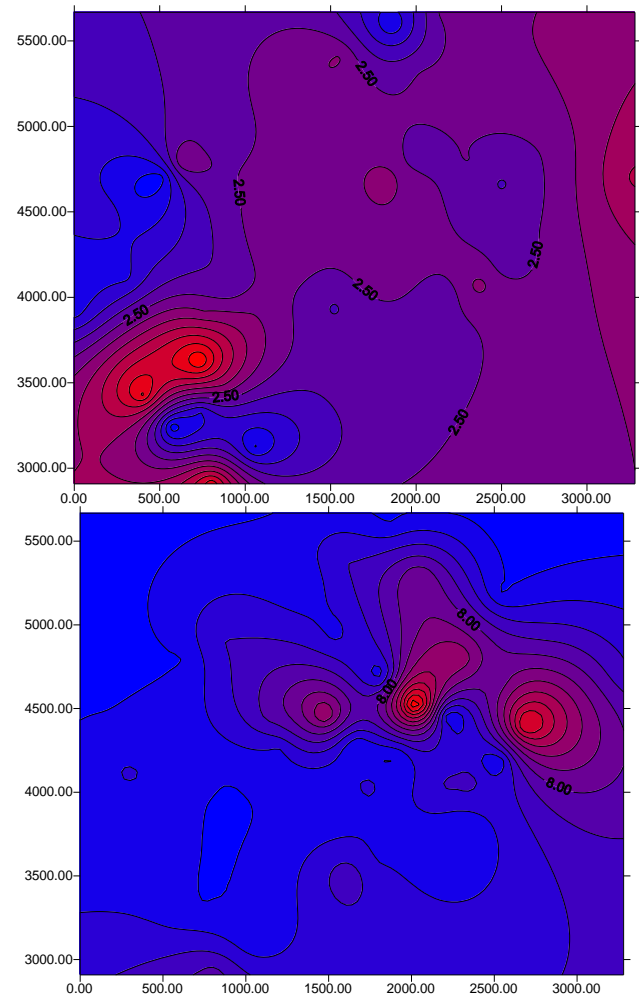

(a)

(b)

Figure 5. a: The Contour Map of M10 b: The Contour Map of Sandstone under M10 




Figure 6. Log-log Plot of Coal Thickness(h) and Area(>=h)

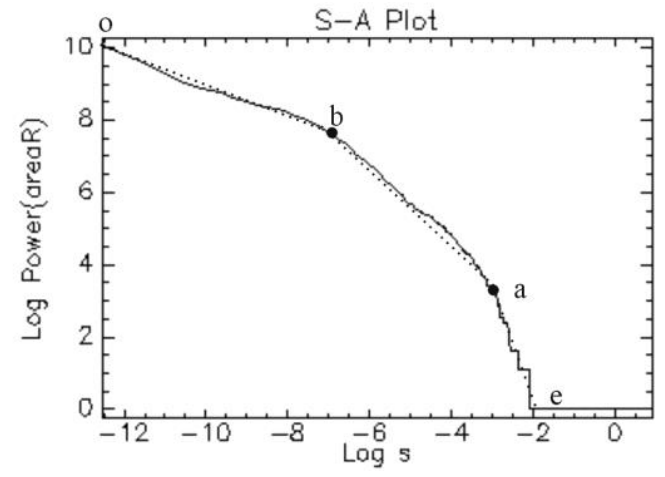

Figure 7. Log-log Plot of Energy(s) and Area(>=s)
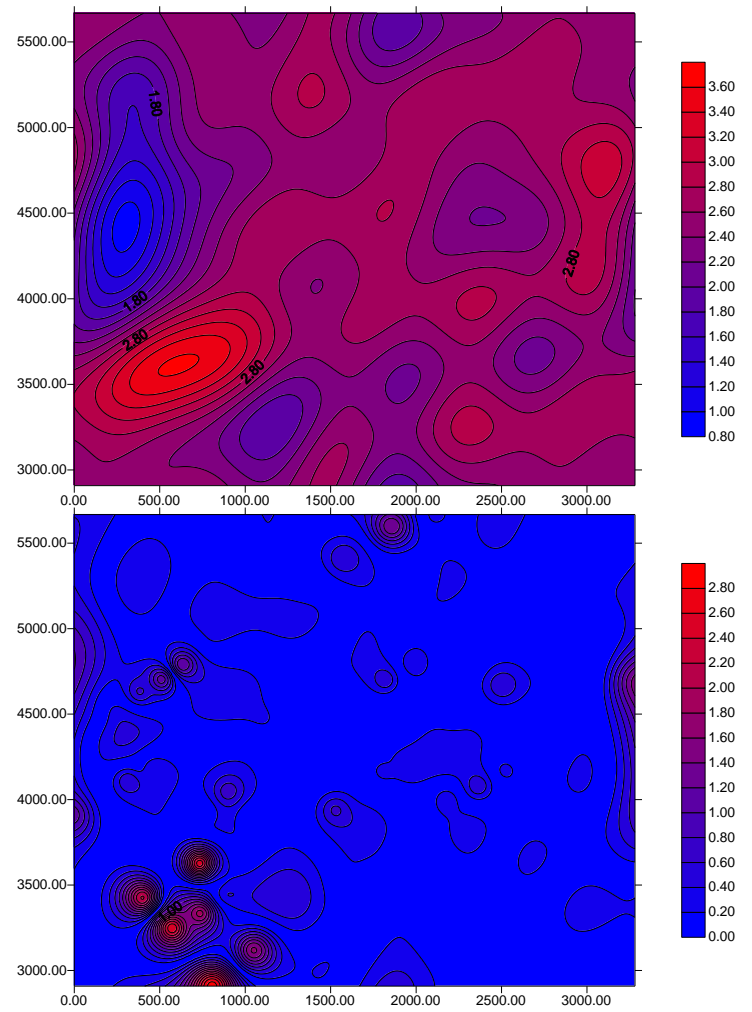

(a)

(b)

Figure 8. a: The Background Map of M10 by S-A b: The Abnormal Map of M10 by S-A 


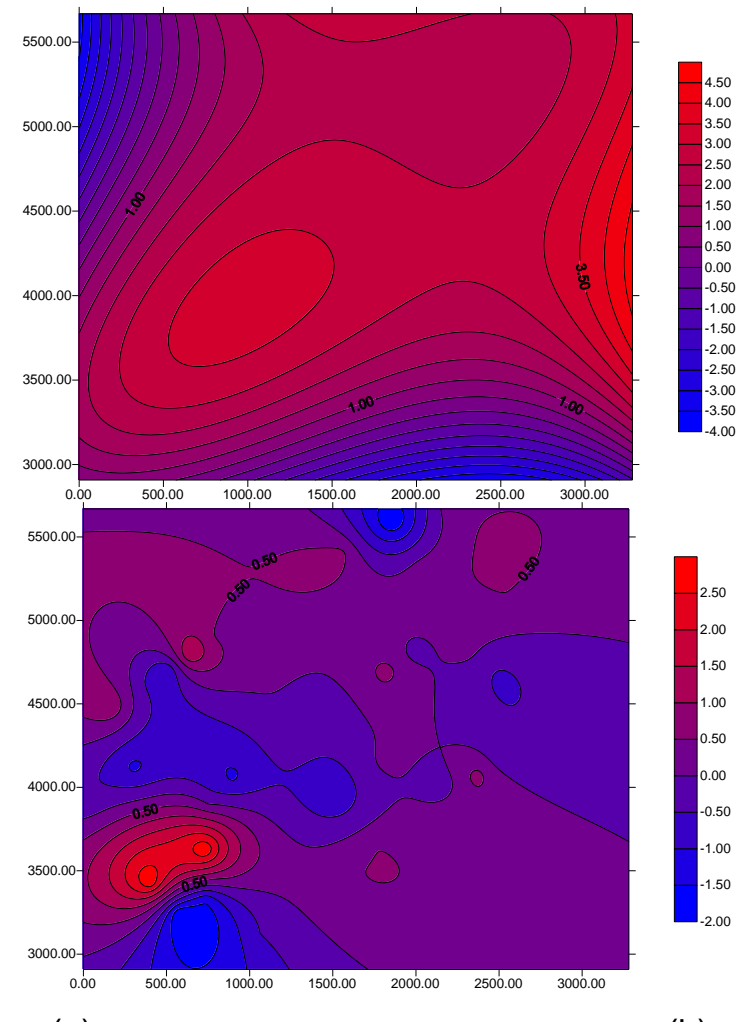

(a)

(b)

\section{Figure 9. a: The Trend Map of M10 Thickness b: The Residual Map of M10} Thickness

Figure 9a is the trendy map of M10 by trendy surface analysis, showing two enrichment centers from west to east which also satisfies the paleo-topography in the Figure 5b wholly but not so clearly and accurately compared with Figure 8a. The residual map shows the secondary change of M10 in the south and north along west to east with a greater difference compared with Figure 8a.

\section{Conclusions}

This paper confirms whether the resources of coal or the thickness of coal are following fractal and multi-fractal distribution. The contour map of coal thickness is decomposed by S-A based on the principle of self-similarity, the result of background map of M10 is coincident with the underlying sandstone which represents the ancient depositional environment from the view of depositional law, and the residual map of M10 is also suitable to show the secondary change of coal thickness. The law of M10 change is clearer and more reasonable compared to the depositional background when compared with the results from trend surface analysis.

The reason is that the data of coal thickness follows multi-fractal distribution, but the trend analysis is based on multiple regression model which is hard to fit because the big deviation caused by the outliers. For the complicated mineralization system, West and Shlesinger's [15] study suggests that the sampled data from them varies from normal to logarithmic normal distribution and then to pareto distribution along with the system becoming more complex, showing strong characteristic of fractal and multi-fractal, the traditional method of data processing is unreasonable because of the unmeet of stationary ${ }^{[1,6]}$. So the processing and analysis of geological data is favor of multi-fractal model for the self-similarity result from the nonlinear dynamic geology process. 


\section{Acknowledgments}

I would like to express my gratitude to all those who helped me during the writing and publishing of this thesis and this thesis is sponsored by NSFC (NO.: 41372368), NSFC of Anhui(NO.:1408085MD761).

\section{References}

[1] Cheng Qiuming, "Singularity of Mineralization and Multifractal Distribution of Mineral Deposits", Bullet in of Mineralogy, Petrology and Geochemistry, vol.27,no.3,(2008),pp.298-305

[2] Renguang Zuo,Qiuming Cheng,Frederik P. Agterberg,Qinglin Xia, "Evaluation of the uncertainty in estimation of metal resources of skarn tin in Southern China",Ore Geology Reviews,(2009),pp.415-422

[3] Du Wen feng,Peng Suping, "Coal seam thickness predication with geostatistic", Chinese journal of rock mechanics and engineering,VOL, 29, NO. S1, (2010),PP.2762-2767

[4] He Qing,Dong Kangqian,Ynag Jun,Evalution on coal mining condition and fully mechanized mining suitability of Shagoucha coal field,Journal of Xi'an university of science and technology,VOL,33,NO.6,(2013),PP.662-668

[5] Cao Daiyong,Liu Qinfu,Peng Suping,He Rixing,Mu Xuanshe,Shi Xianzhong,Zhang Shouren, "Quantitative Analysis on The Thickness Variation of NO.2-1 Coal Seam in Chaohua Mine Field", Coal Geology\& Exploration,V0,.26,NO.5,(1998),pp.28-32

[6] Cheng Qiuming, "Singularity-Generalized Self-Similarity-Fractal Spectrum(3S) Models", Earth Science-Journal of China University of Geosciences, VOL.31.No.3,(2006),pp.337-348.

[7] $\mathrm{Li}$ Qingmou, "Multifractal-kriging interpolation method",Advances in earth science,vol,20,no,2,(2005),pp.248-256

[8] Liu Y, Q. Cheng,Q.Xia,X.Wang, "Identification of REE mineralization-related geochemical anomalies using fractal/multifractal methods in the Nanling belt,South China",Environ Earth Sci,vol,72,(2014),pp.5159-5169

[9] Yang Mengda, “Coal Geology”,Beijing:Coal Industry Press,(2000).

[10] West BJ,Shlesinger M, "The noise in natural phenomena".AmSci 78,pp.40-50,(1990).

[11] Liu Hengqiu,Liu Qinfu,Peng Suping,Tan Rujiao,Fu Zheng, "Evolvement characteristic of sand bodies in the fourth coal-bearing interval and its control on coal thickness,Huainan coalfield",Coal Geology\&Explortion,vol,33,no,1,(2005),pp.7-10

[12] Xu Dejin,Hu Baolin,Wang Hongzhi, "Application of Trend Surface Analysis to Magma tic Rock Affected to Seam", Coal Science and Technology, vol,37,no.2,(2009),pp.108-110

[13] Li Xiaohui,Yuan Feng,JiaCai,Zhang Mingming,Zhou Taofa, "Contrastive study of S-A multifractal filtering method based on inverse distance weighted and Kriging interpolation",Science of Surveying and Mapping, vol.37 no.3,(2012),pp. 87-89

[14] Shuyun Xie,Qiuming Cheng,Gao Chen,Zhijun Chen, and Zhengyu Bao, "Application of local singularity in prospecting potential oil/gas Targets", Nonlinear Processes in Geophysics,(2007):285-292

[15] Alireza Almasi,Alireza Jafarirad,Peyman Afzal, "Prospecting of gold,mineralization in Saqez area (NW Iran) using geochemical, geophysical and geological studies based on multifractal modelling and principal component analysis Mana Rahimi”,Arab J Geosci,vol,8,(2015),pp.5935-5947

\section{Authors}

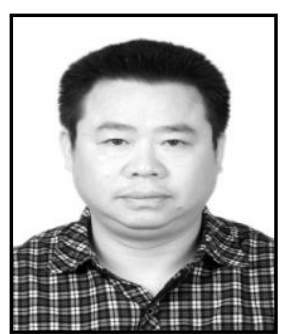

Liu Xing, male, born in 1974, vice processor, doctor of Philosophy, the study interesting is GIS and hyper-spectrum remote sensing and them application in geology. 
International Journal of Signal Processing, Image Processing and Pattern Recognition Vol. 10, No. 4 (2017) 